\title{
Generation of acoustic rogue waves in dusty plasmas through three-dimensional particle focusing by distorted waveforms
}

\author{
Ya-Yi Tsai, Jun-Yi Tsai and Lin I*
}

\begin{abstract}
Rogue waves-rare uncertainly emerging localized events with large amplitudes-have been experimentally observed in many nonlinear wave phenomena, such as water waves ${ }^{1-6}$, optical waves $^{7,8}$, second sound in superfluid He II (ref. 9) and ion acoustic waves in plasmas ${ }^{10}$. Past studies have mainly focused on one-dimensional (1D) wave behaviour through modulation instabilities $1,3-5,7,11$, and to a lesser extent on higher-dimensional behaviour $5,6,8,11,12$. The question whether rogue waves also exist in nonlinear 3D acoustic-type plasma waves, the kinetic origin of their formation and their correlation with surrounding 3D waveforms are unexplored fundamental issues. Here we report the direct experimental observation of dust acoustic rogue waves in dusty plasmas and construct a picture of 3D particle focusing by the surrounding tilted and ruptured wave crests, associated with the higher probability of low-amplitude holes for rogue-wave generation.
\end{abstract}

Modulation instability (MI) which makes the wave modulation envelope unstable has been well accepted as a mechanism for rogue-wave or envelope soliton generation in systems governed by nonlinear equations, such as the nonlinear Landau-Ginzburg or Schrödinger equations $s^{1,3-5,7,11,12}$. On the other hand, recent studies in nonlinear water, chemical and dust acoustic waves, also demonstrated that MI causes $3 \mathrm{D}$ waveform undulation, rupture and reconnection; and generates opposite singular objects, lowamplitude hole (LAH) filaments coinciding with defect trajectories, where wave amplitudes are null and phases are undefined ${ }^{13-16}$.

In a $1 \mathrm{D}$ longitudinal density wave constituted by particles, from the wave-particle interaction view, waveforms determine longitudinal wave fields, which affect particle motion, and in turn waveform evolution ${ }^{17,18}$. A recent experimental study of $3 \mathrm{D}$ unstable dust acoustic waves (DAWs) showed that the additional transverse (normal to the wave propagation direction) wave fields from distorted and ruptured waveforms around LAHs are further key factors affecting particle motion, and in turn LAH evolution ${ }^{19}$. Certainly, it is intriguing to ask whether and how rogue wave event (RWE) formation can also be understood through wave-particle interactions in strongly undulated regions, by correlating opposite singular objects-RWEs and LAHs-with their surrounding 3D waveforms and particle motion.

A DAW, associated with the low-frequency longitudinal oscillation of negatively charged dust particles in gaseous plasmas, is a fundamental nonlinear acoustic-type density wave, governed by modulation-type nonlinear dynamical equations $\mathrm{s}^{20-22}$. It can be self-excited through the interplay of dust inertia, screened Coulomb interactions and ion streaming ${ }^{23}$. Studies on shocks ${ }^{24-26}$, solitary waves ${ }^{27}$, wave breaking ${ }^{18}$, oscillons ${ }^{28}$, defects and LAHs (refs 15,16,19,29) are the few examples giving experimental evidence of the ubiquitous behaviour in many other nonlinear media. The advantages of direct video imaging large-area dust density evolution and tracking individual particle motion at the discrete level also make it a good platform to construct an EulerianLagrangian picture as a means of understanding dynamics in nonlinear density wave systems ${ }^{18,19,28}$. Nevertheless, RWEs have been demonstrated theoretically only in 1D dust acoustic waves ${ }^{22}$.

The experiment is conducted in a cylindrical radiofrequency (rf) dusty-plasma system, as sketched in Fig. 1a (also see Methods) ${ }^{16}$. Figure $1 \mathrm{~b}$ shows a typical temporal waveform of $n_{\mathrm{d}}$, the normalized local dust density, in the disordered state of the self-excited downward propagating DAW. The irregular amplitude modulation evidences MI and causes the broadening of the fundamental and higher harmonic peaks in its power spectrum (Fig. 1c).

Figure $1 \mathrm{~d}$ shows the histogram of the wave height $H$ measured from 12,000 images. As commonly used for oceanic RWEs, the stretched tail beyond $2 H_{\mathrm{s}}$ signifies RWEs, where $H_{\mathrm{s}}(=2)$ is the significant wave height, defined as the average of the highest third of all wave heights ${ }^{1,2}$. Figure 1e shows the highly localized and randomly distributed RWEs in the xyt space over 120 wave cycles. The averaged wavelength $\lambda$ and wave period $\tau_{0}$ are $1 \mathrm{~mm}$ and $32 \mathrm{~ms}$, respectively.

Figure 1f shows typical temporal $n_{\mathrm{d}}$ waveforms around RWEs (RWEs A to $\mathrm{C}$ in Fig. 1e). By resetting the crests $2 H_{\mathrm{s}}$ in height (RWEs) and $1.3 H_{\mathrm{s}}$ in height at $\tau=0$, Fig. $1 \mathrm{~g}$ shows the evolutions of the averaged modulation envelope height (vertical width) $\left\langle H_{\mathrm{E}}\right\rangle$ over the waveforms before and after these events (see Methods for event numbers). On average, the sharp increase in amplitude during one wave cycle to reach the RWE peak is preceded by a slow MI-induced amplitude growth lasting five wave cycles, whereas the $1.3 H_{\mathrm{s}}$ crest has a warning of less than two wave cycles. Note that an individual RWE is not necessarily preceded by a monotonically increasing wave height (Fig. 1f and Supplementary Information), and other higherdimensional effects need to be considered for RWE formation.

MI also causes undulation of the $3 \mathrm{D}$ waveform, as shown in the $x t$ and $y t$ planes of Fig. 2 a (also see refs 15,16 , for a typical undulated waveform). The arrow in Fig. 2a indicates a highly localized RWE on the top $x y$ plane. LAHs (defects) labelled by crosses are located at the vertices of pitchfork-like structuress with connected or broken arms, where the wave amplitudes are null and the phases are undefined ${ }^{15,16}$. Figure $2 \mathrm{~b}, \mathrm{c}$ shows 3D plots of LAH filaments (defect trajectories) in the $x y t$ space surrounding two typical RWEs.

How do RWEs and LAH filaments correlate statistically? The contour plots of Fig. $2 \mathrm{~d}$ depict the distribution function $P(r, \tau)$, the probability density of finding LAHs with spatial and temporal 
a

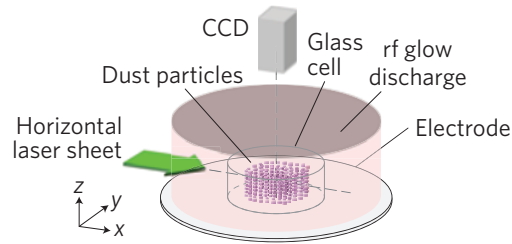

b

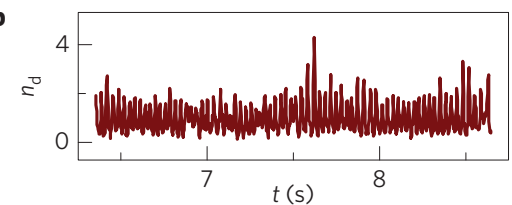

c

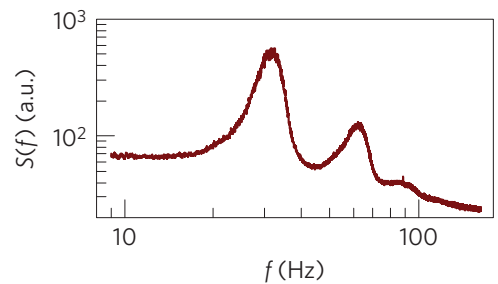

d

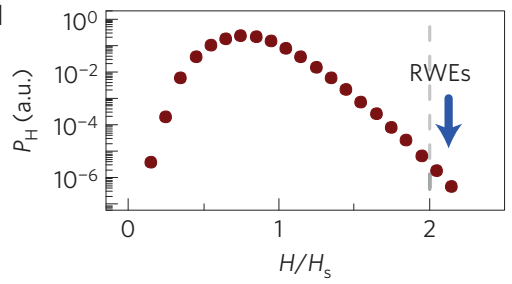

e

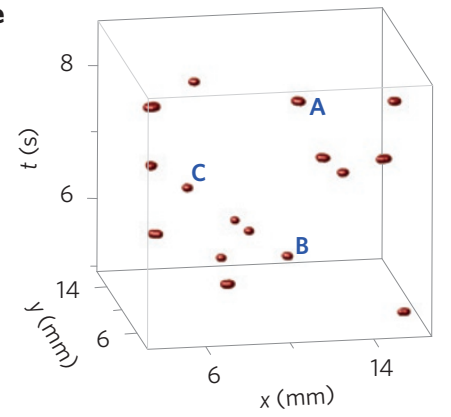

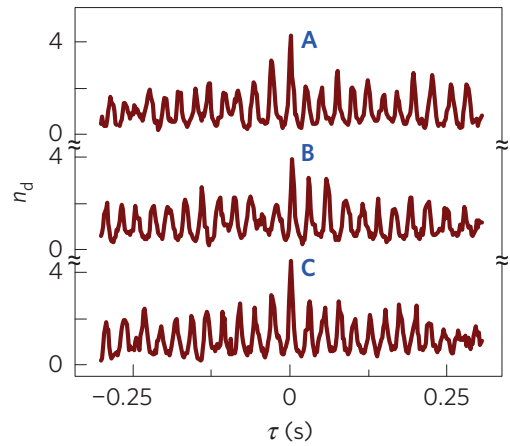

g

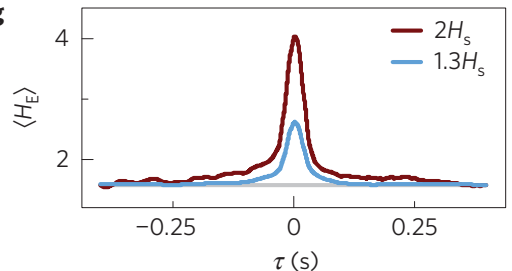

Figure 1 | Experimental system and information evidencing RWEs. a, Sketch of the experimental system. The laser sheet and CCD can also be rotated

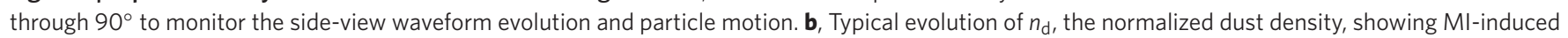

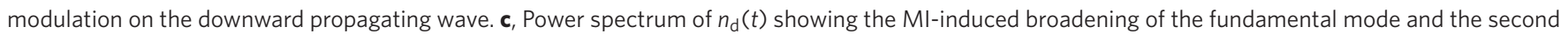
harmonic, peaked at 31.5 and $63 \mathrm{~Hz}$, respectively. $\mathbf{d}$, Histogram of the ratio of wave height $H$ to significant wave height $H_{\mathrm{s}}$, showing the existence of RWEs at the stretched tail end $\left(H \geq 2 H_{s}\right)$. e, Randomly distributed RWE locations in the xyt space over 120 wave cycles. f, Typical $n_{d}$ evolutions measured at different locations in which the RWE peak times have been reset at $\tau=0 . \mathbf{g}$, Temporal evolutions of the envelope height $\left\langle H_{\mathrm{E}}\right\rangle$, averaged over $\mathrm{crests} 2 \mathrm{H}_{\mathrm{S}}$ in height (RWEs) and crests $1.3 \mathrm{H}_{\mathrm{s}}$ in height at $\tau=0$. The sharp rise to reach the RWE peak tends to be preceded by a few crests with a slowly growing

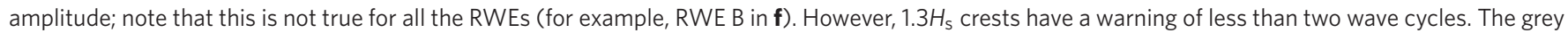

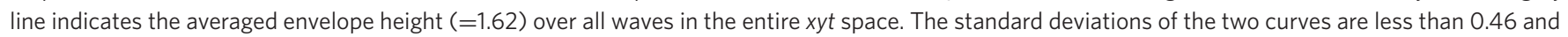
0.37 for RWEs and crests $1.3 H_{\mathrm{s}}$ in height, respectively, for the entire curves (see Supplementary Information).

separations $r$ and $\tau$, respectively, from crests with $H=2,1.3$ and $1 H_{s}$, where negative $\tau$ indicates that LAHs appear before the large crest. Clearly, in the top panel of Fig. $2 \mathrm{~d}$, from $4 \tau_{0}$ before the RWE, LAHs start to have a slightly higher probability of occurrence in a cylindrical shell $1.5 \lambda$ in radius than the average probability in space $(=0.019$, coded in white). The probability reaches its maximum $(P=0.026)$ in a ring approximately $0.55 \lambda$ in radius $0.4 \tau_{0}$ before the RWE. However, the blue LAH peak decreases in height and diminishes in extent for 1.3 and $1 H_{s}$ events, respectively. Obviously, the preceding neighbouring $\mathrm{LAH}$ filaments are important for emerging giant crests.

The decreasing likelihood (coded by shaded orange) of LAHs in a cylinder $0.3 \lambda$ in radius approaching an RWE is consistent with the slow growth of averaged wave height. However, what are the roles of the surrounding LAH filaments before an RWE?

Let us construct a kinetic picture based on wave-particle interactions. Our previous experiment correlating particle motion and waveform evolution shows that, in an ordered travelling plane DAW, particles interact strongly with and oscillate in the longitudinal wave field ${ }^{18,19}$. Particles are negatively charged and the wave potential field for a test particle thereby oscillates in phase with the washboard-type particle density field ${ }^{18,19}$. As shown in Fig. 3a, in a downward travelling plane wave, the accumulation and depletion of particles, caused by strong deceleration (compression) and acceleration (rarefaction) of the upward moving particles entering the crest front and leaving the crest rear, produced by longitudinal forces from the crest towards the neighbouring troughs, sustain and determine evolution of the waveform. The above effects are stronger for larger amplitude crests ${ }^{18,19}$.

The above $1 \mathrm{D}$ picture is complicated for an undulated waveform. Because forces are normal to the contour lines of dust density, a tilted wave crest surface and a non-uniform $n_{\mathrm{d}}$ distribution along a crest both introduce additional transverse forces ${ }^{19}$. Sequential snapshots of the $n_{\mathrm{d}}(x, z)$ contour plots in Fig. $3 \mathrm{~b}$ show an example of a downward moving undulated waveform, measured from images illuminated by a vertical laser sheet. Here $z$ is the vertical axis. The vectors indicate the tilted forces. A few typical particle trajectories are also plotted in the lower panel. Obviously, for the waveform around the LAH labelled by a cross at the broken end of crest C2, the leftward force from the rear of the tilted crest $\mathrm{C} 1$ and the rightward diverging field from the broken end of $\mathrm{C} 2$ enhance focusing of particles from $\mathrm{C} 1$ and $\mathrm{C} 2$ rear into high crest region $\mathrm{H}$. The broken end of $\mathrm{C} 2$ also provides an additional supply of particles moving towards the upper right direction for focusing. The above processes greatly enhance the accumulation of particles and the growth in amplitude for region $\mathrm{H}$ from -7.5 to $0 \mathrm{~ms}$. Figure $3 \mathrm{c}$ gives a further view of the undulated waveform around the RWE in the $x \tau$ space, measured at $z=0.5 \mathrm{~mm}$.

On the basis of the above picture, the generation of an RWE preceded by the formation of surrounding LAH filaments can be understood. Figure 4a,b, and the video in the Supplementary Information depict the $3 \mathrm{D}$ structure of wave crest surfaces and LAH filaments in the $x y t$ space surrounding the RWE in Fig. 2a. Similar to the previous study ${ }^{16}$, LAH filaments are accompanied by surrounding ruptured and tilted crest surfaces through MI-induced undulation, which appear as broken and tilted crests, respectively, in the two different vertical planes $\left(x_{1} t\right.$ and $\left.x_{2} t\right)$ intersecting an RWE in Fig. 4c,d. Tilted and broken crests before the RWE in Fig. 4c, similar to those of Fig. 3, induce particle focusing and accumulation in the crest front, manifested by narrowing and growth of the high-amplitude clump in $\mathrm{C} 1$ to the RWE in C3. In another vertical $x_{2} t$ plane, the opposite two ends of the leading broken crest $\mathrm{C} 2$ provide particle supply and focusing, although their leading crest $\mathrm{C} 1$ is not tilted (Fig. 4d). Another example manifesting 
a
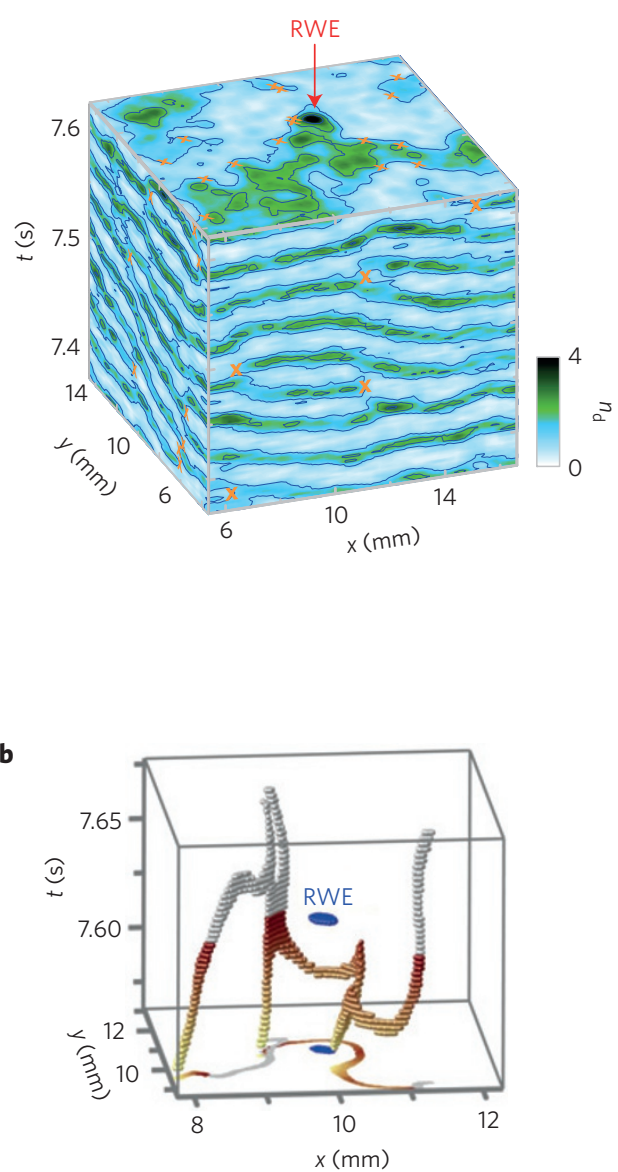

d

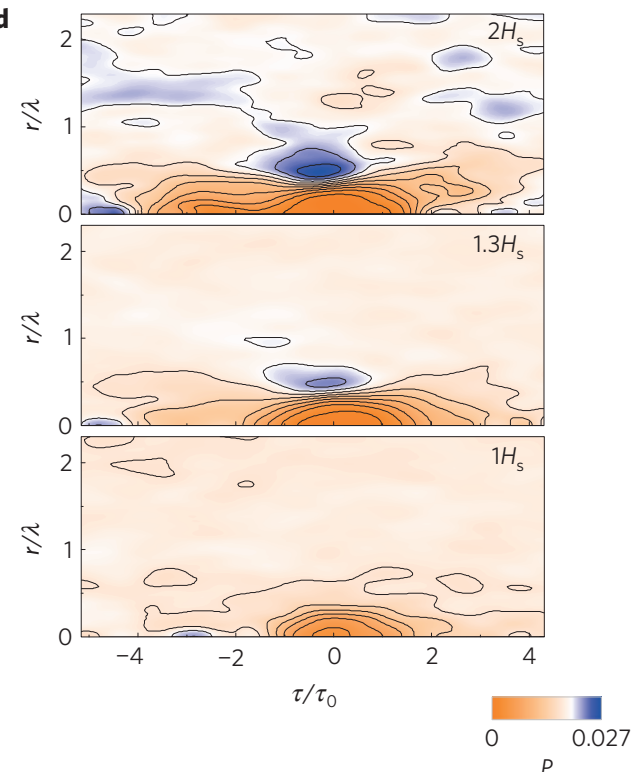

c

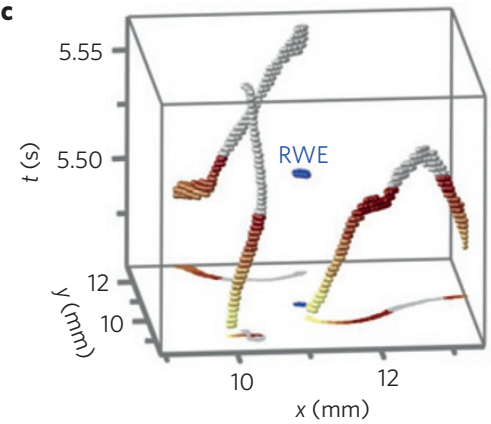

Figure 2 | Undulated waveforms and LAHs nearby RWEs. a, Distribution of $n_{d}$ on $x y, x t$ and $y$ t planes showing the typical undulated waveform. The arrow labels the RWE (the dark region), localized on the top xy plane. The crosses indicate the locations of the defects (LAHs) where wave amplitudes are null and phases are undefined. b,c, Irregular hole filaments surrounding two typical RWEs, in the xyt space, with their projections on the floor. The filaments before RWEs are indicated by shades of brown. $\mathbf{d}$, Contour plots of the probability density of finding LAHs with spatial and temporal separations $r$ and $\tau$, respectively, from crests with different $H$, where $\lambda$ and $\tau_{0}$ are the averaged wavelength and period, respectively. The average probability over the entire space of the top panel (0.019, see Supplementary Information) is indicated by white, and the higher (lower) probabilities are indicated by shades of blue (orange). The very low probability of LAHs in the centre cylinder $(r<0.3 \lambda)$ and the higher probability of LAHs in the surrounding ring before RWE are important for RWE formation. The blue peak decreases in height and diminishes in extent for crests with $H=1.3$ and $1.0 H_{s}$, respectively.

particle focusing and enhanced RWE formation is shown in the Supplementary Information.

At the rear of the crest of an RWE clump, diverging wave fields cause defocusing of exiting particles (see particle trajectories in Fig. 3b). This decreases the particle accumulation in the trailing crest front and the consequent crest amplitude (also see the drop in wave amplitude following the RWEs in Fig. 1f).

In conclusion, using self-excited DAWs as a platform, we have demonstrated the first direct observation of RWEs in self-excited 3D longitudinal plasma density waves, and constructed a wave-particle interaction picture for RWE formation, by correlating RWEs, LAHs, their surrounding 3D undulated waveforms, and individual particle motion. It is found that, in addition to the slow leading growth in amplitude, RWEs are preceded by a higher probability of surrounding LAH filaments, associated with distorted waveforms. In addition to the longitudinal wave field affecting particle accumulation and depletion in the crest front and rear, respectively, transverse forces from the leading tilted or ruptured crests nearby LAH filaments assists $3 \mathrm{D}$ focusing of particles entering the trailing crest front, for generating spatiotemporally localized RWEs.

Note that a recent delayed time series analysis using single local dynamical variables for sea and optical waves found that RWEs do not necessarily appear without a short phase warning ${ }^{30}$. Our study sheds light on the subject of RWE formation and its short phase statistical precursors for longitudinal density waves in plasmas or gaseous media.

\section{Methods}

Methods and any associated references are available in the online version of the paper.

Received 3 September 2015; accepted 15 January 2016; published online 29 February 2016

\section{References}

1. Kharif, C., Pelinovsky, E. \& Slunyaev, A. Rogue Waves in the Ocean (Springer, 2009).

2. Akhmediev, N. \& Pelinovsky, E. Editorial-Introductory remarks on "Discussion Debate: Rogue waves-Towards a unifying concept?" Eur. Phys. J. Spec. Top. 185, 1-4 (2010).

3. Chabchoub, A., Hoffman, N. P. \& Akhmediev, N. Rogue wave observation in a water wave tank. Phys. Rev. Lett. 106, 204502 (2011).

4. Onorato, M., Osborne, A. R., Serio, M. \& Cavaleri, L. Modulational instability and non-Gaussian statistics in experimental random water-wave trains. Phys. Fluids 17, 078101 (2005) 
a

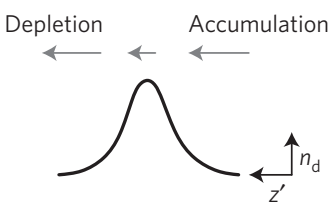

b

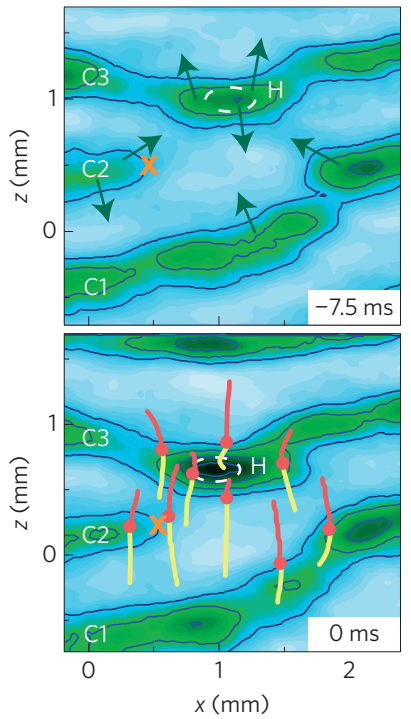

c
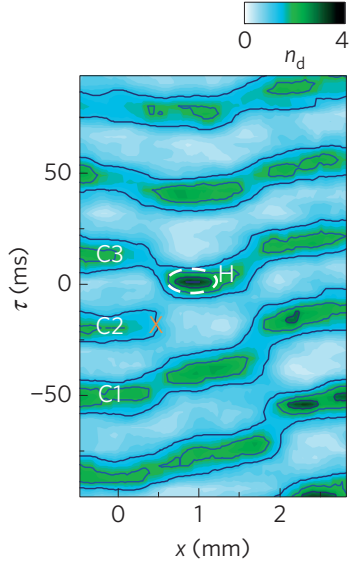

Figure $\mathbf{3}$ | Wave-particle interaction picture showing an RWE emerging through particle focusing provided by leading undulated and ruptured crests. a, Cartoon (with the arrow length indicating particle speed) showing the deceleration (acceleration) of upward moving particles entering (leaving) a crest, leading to particle compression (rarefaction), subsequent particle accumulation (depletion) in the crest front (rear), and wave crest advance, for a 1D plane wave, where $z^{\prime}$ is the vertical coordinate moving with the downward (along $-z$ ) travelling wave. $\mathbf{b}$, Sequential $n_{d}(x, z)$ (side-view distribution) plots showing downward moving undulated waveforms. The crosses indicate the LAH locations. Yellow and red trajectories correspond to the $7.5 \mathrm{~ms}$ trajectories of a few typical dust particles in the wave moving frame, before and after current particle positions represented by dots, respectively. The arrows indicate the wave field forces normal to the $n_{d}$ contour lines for dust particles, leading to the focusing (defocusing) of particles entering (leaving) the high-amplitude region $\mathrm{H}$. It causes the rapid growth in the wave height of the circled region $\mathrm{H}$, with lower amplitude of the trailing crest. c, Contour plot of $n_{d}$ in the $x \tau$ plane, measured at $z=0.5 \mathrm{~mm}$, showing the waveform around the same RWE as in $\mathbf{b}$.

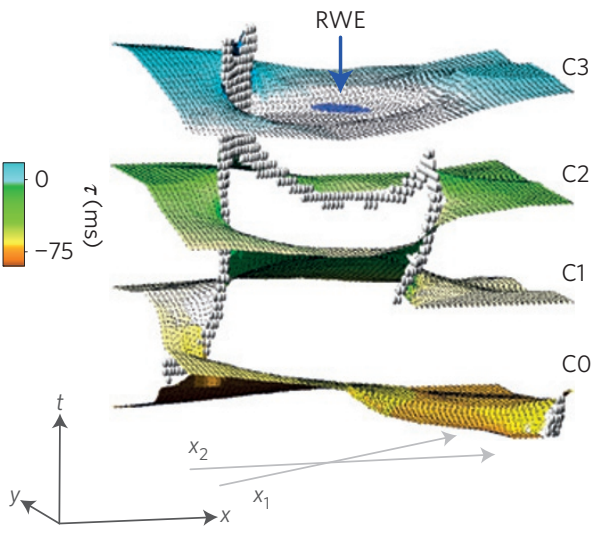

c

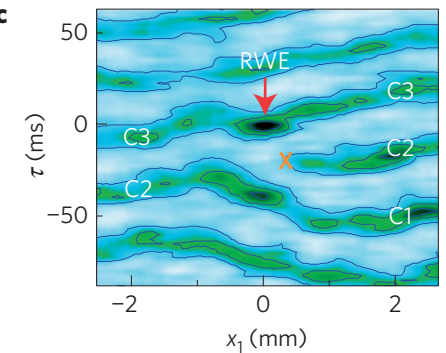

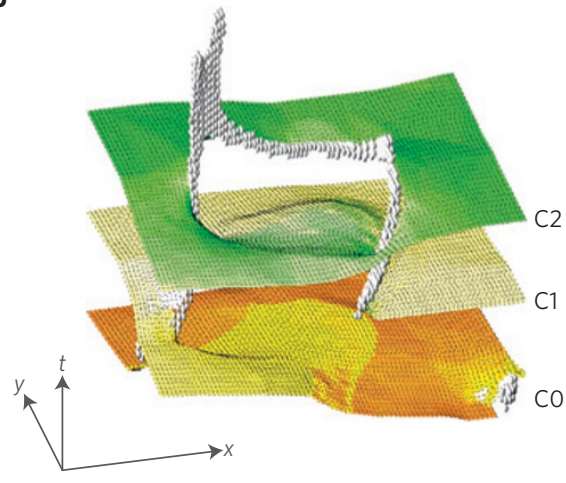

d

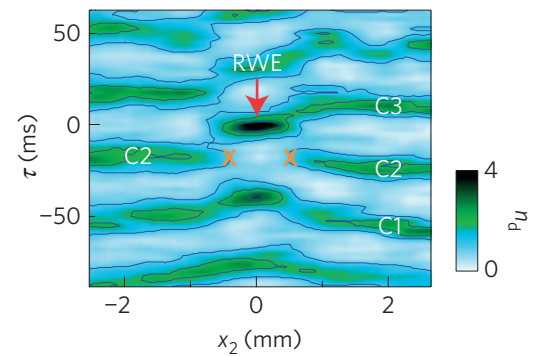

Figure 4 | Undulated 3D waveform leading to 3D particle focusing and REW generation. a, Three-dimensional waveform ( $2 \mathrm{~mm}$ in width) surrounding an RWE in the blue disc region indicated by the arrow, on the top crest surface. Only wave crests and LAH filaments (grey dots) are plotted. The dark areas correspond to the bottom sides of tilted crest surfaces. $\mathbf{b}$, Same plot as $\mathbf{a}$, omitting the top crest $\mathrm{C} 3$, from a different angle to give a better view of the leading waveforms. Crest C2 is ruptured along a curved line. The ruptured tongue is bent downwards (appears as the dark surface between $\mathrm{C} 2$ and $\mathrm{C} 1$ in a and the green tongue in $\mathbf{b}$ ), and reconnected with another ruptured edge of $\mathrm{C} 1$. A similar rupture and reconnection process also occurs between $\mathrm{C} 1$ and $\mathrm{CO}$. The $\mathbf{C}$-shaped curved broken edge of $\mathrm{C} 2$ and the tilted green tongue in $\mathbf{b}$ provide additional particle supply and transverse forces for 3D particle focusing and RWE generation. $\mathbf{c}, \mathbf{d}$, Contour plots of $n_{d}$ in two different vertical planes $x_{1} t$ and $x_{2} t$ intersecting the RWE in a, again manifesting the above 3D particle focusing effects caused by the distorted waveform preceding the RWE. 
5. Shats, M., Punzmann, H. \& Xia, H. Capillary rogue waves. Phys. Rev. Lett. 104, 104503 (2010).

6. Xia, H., Maimbourg, T., Punzmann, H. \& Shats, M. Oscillon dynamics and rogue wave generation in Faraday surface ripples. Phys. Rev. Lett. 109, $114502(2012)$.

7. Solli, D. R., Ropers, C., Koonath, P. \& Jalali, B. Optical rogue waves. Nature 450, 1054-1057 (2007).

8. Montina, A., Bortolozzo, U., Residori, S. \& Arecchi, F. T. Non-Gaussian statistics and extreme waves in a nonlinear optical cavity. Phys. Rev. Lett. 103, 173901 (2009).

9. Ganshin, A. N., Efimov, V. B., Kolmakov, G. V., Mezhov-Deglin, L. P. \& McClintock, P. V. E. Observation of an inverse energy cascade in developed acoustic turbulence in superfluid helium. Phys. Rev. Lett. 101, 065303 (2008).

10. Bailung, H., Sharma, S. K. \& Nakamura, Y. Observation of peregrine solitons in a multicomponent plasma with negative ions. Phys. Rev. Lett. 107, 255005 (2011).

11. Onorato, M., Osborne, A. R. \& Serio, M. Modulational instability in crossing sea states: A possible mechanism for the formation of freak waves. Phys. Rev. Lett. 96, 014503 (2006).

12. Shukla, P. K., Kourakis, I., Eliasson, B., Marklund, M. \& Stenflo, L. Instability and evolution of nonlinearly interacting water waves. Phys. Rev. Lett. 97, 094501 (2006).

13. Shani, I., Cohen, G. \& Fineberg, J. Localized instability on the route to disorder in Faraday waves. Phys. Rev. Lett. 104, 184507 (2010).

14. Qiao, C., Wang, H. \& Ouyang, Q. Defect-mediated turbulence in the Belousov-Zhabotinsky reaction. Phys. Rev. E 79, 016212 (2009).

15. Chang, M. C., Tsai, Y. Y. \& I, L. Observation of 3D defect mediated dust acoustic wave turbulence with fluctuating defects and amplitude hole filaments. Phys. Plasma 20, 083703 (2013).

16. Tsai, Y. Y. \& I, L. Observation of self-excited acoustic vortices in defect-mediated dust acoustic wave turbulence. Phys. Rev. E 90, 013106 (2014).

17. Chen, F. F. Introduction to Plasma Physics Ch. 7 (Plenum Press, 1974).

18. Teng, L. W., Chang, M. C., Tseng, Y. P. \& I, L. Wave-particle dynamics of wave breaking in the self-excited dust acoustic wave. Phys. Rev. Lett. 103, 245005 (2009).

19. Tsai, J. Y., Tsai, Y. Y. \& I, L. Wave-particle dynamics of waveform and defect evolutions in undulated nonlinear self-excited dust acoustic waves. Phys. Plasmas 22, 013701 (2015).

20. Rao, N. N., Shukla, P. K. \& Yu, M. Y. Dust-acoustic waves in dusty plasmas. Planet. Space Sci. 38, 543-546 (1990).
21. Amin, M. R., Morfill, G. E. \& Shukla, P. K. Modulational instability of dust acoustic and dust-ion-acoustic waves. Phys. Rev. E 58, 6517-6523 (1998).

22. Moslem, W. M., Sabry, R., El-Labany, S. K. \& Shukla, P. K. Dust-acoustic rogue waves in a nonextensive plasma. Phys. Rev. E 84, 066402 (2011).

23. Kaw, P. \& Singh, R. Collisional instabilities in a dusty plasma with recombination and ion-drift effects. Phys. Rev. Lett. 79, 423-426 (1997)

24. Samsonov, D. et al. Kinetic measurements of shock wave propagation in a three-dimensional complex (dusty) plasma. Phys. Rev. E 67, 036404 (2003).

25. Heinrich, J., Kim, S.-H. \& Merlino, R. L. Laboratory observations of self-excited dust acoustic shocks. Phys. Rev. Lett. 103, 115002 (2009).

26. Shukla, P. K. \& Eliasson, B. Colloquium: fundamentals of dust-plasma interactions. Rev. Mod. Phys. 81, 25-44 (2009).

27. Bandyopadhyay, P., Prasad, G., Sen, A. \& Kaw, P. K. Experimental study of nonlinear dust acoustic solitary waves in a dusty plasma. Phys. Rev. Lett. 101, 065006 (2008)

28. Zhdanov, S., Schwabe, M., Rath, C., Thomas, H. M. \& Morfill, G. E. Wave turbulence observed in an auto-oscillating complex (dusty) plasma. Europhys. Lett. 110, 35001 (2015).

29. Williams, J. D. Evolution of frequency clusters in the naturally occurring dust acoustic wave. Phys. Rev. E 89, 023105 (2014).

30. Birkholz, S., Bree, C., Demircan, A. \& Steinmeyer, G. Predictability of rogue events. Phys. Rev. Lett. 114, 213901 (2015).

\section{Acknowledgements}

This work is supported by the Ministry of Science and Technology, Taiwan, under contract No. MOST 102-2112-M-008-017-MY3.

\section{Author contributions}

Y.-Y.T. and J.-Y.T. performed the experiment and data analysis. Y.-Y.T. and L.I. jointly wrote the paper. All authors took part in discussion of the results and contributed to the manuscript. L.I. supervised the entire work

\section{Additional information}

Supplementary information is available in the online version of the paper. Reprints and permissions information is available online at www.nature.com/reprints.

Correspondence and requests for materials should be addressed to L.I.

\section{Competing financial interests}

The authors declare no competing financial interests. 


\section{Methods}

The experiment is conducted in a cylindrical rf dusty-plasma system, as shown in Fig. 1a, similar to our previous experiment ${ }^{16}$. A hollow cylindrical thin glass trap of inner diameter $32 \mathrm{~mm}$ and height $18 \mathrm{~mm}$ is placed on the centre of the bottom electrode to confine a dusty plasma consisting of polystyrene particles $(5 \mu \mathrm{m}$ in diameter, $6.9 \times 10^{-11} \mathrm{~g}$ in mass, $6 \times 10^{4} \mathrm{~cm}^{-3}$ in density), charged with about ten thousand electrons per particle. The weakly ionized glow discharge $\left(n_{\mathrm{e}} \sim 10^{9} \mathrm{~cm}^{-3}\right)$ supporting the self-excited downward propagating (along the $-z$ direction) waveform with parallel straight wave crests can be generated in Ar gas at 220 mtorr using a $14-\mathrm{MHz}$ rf power system at $2.8 \mathrm{~W}$. The estimated Debye length $\lambda_{\mathrm{D}}$ and dust particle charges are of the order of a few tens of micrometres and a few to ten thousands of electrons per particle, respectively. Dust images in a horizontal $x y$ (or vertical $x y)$ plane illuminated by an expanded horizontal laser sheet $(\sim 0.6 \mathrm{~mm}$ in width) are captured by a charge-coupled device (CCD) camera at a sampling rate of $400 \mathrm{~Hz}$. The normalized local dust density, $n_{\mathrm{d}}(x, y, t)=I_{\mathrm{d}}(x, y, t) /\left\langle I_{\mathrm{d}}(x, y)\right\rangle_{t}$, can be obtained by measuring the image brightness $I_{\mathrm{d}}$, coarse grained over a Gaussian weighted circle $\left(0.3 \mathrm{~mm}\right.$ in full-width at half-maximum), where $\left\langle I_{\mathrm{d}}(x, y)\right\rangle_{t}$ is the time average of $I_{\mathrm{d}}(x, y)$ (ref. 17). Individual particle motions in the $x z$ plane are tracked and compared with the side-view waveform to construct a Lagrangian-Eulerian picture for RWE formation.

In our statistical analyses of the wave envelope height curves in Fig. $1 \mathrm{~g}$ and the LAH probability density distributions in Fig. 2d, we used 97 RWE events $2 H_{\mathrm{s}}$ in height, 3,484 crest events $1.3 H_{\mathrm{s}}$ in height, and 46,600 crest events $1.0 H_{\mathrm{s}}$ in height. 